\title{
Assessment of the bearing capacity of welded joints for an arbitrary character of failure
}

\author{
Valery Erofeev ${ }^{1}$, Robert Sharafiev ${ }^{1}$, and Olga Grebenshchikova ${ }^{2, *}$ \\ ${ }^{1}$ Ufa State Petroleum Technological University, Kosmonavtov Str. 1, Ufa, 450062, the Republic of \\ Bashkortostan, Russia \\ ${ }^{2}$ Air Force Military Educational and Scientific Centre Air Force Academy n.a. Prof. N.E. Zhukovsky \\ and Yu.A. Gagarin, Gorodok-11, 1, Chelyabinsk, 454015, Russia
}

\begin{abstract}
In this paper, we propose one of the approaches to assess the load-bearing capacity of metal structures for an arbitrary type of failure based on the use of interpolation-type criteria based on the principle of boundary interpolation. This approach allows for the standardization of defects taking into account their location, sharpness at the top, as well as the properties of the base metal or welded joints. The use of the calculated calculated ratios that have been experimentally confirmed during the testing of welded joints with surface defects allows us to estimate the range of their admissible sizes that do not lead to a decrease in operating loads acting on welded metal structures during their operation, proceeding from ensuring the required level of allowable stresses. This range of permissible defect sizes is determined by a number of parameters characterizing the type of defect, the mechanical properties of the weld metal, the type, stiffness and concentration of the stressed state, the thickness of the metal structures, the operating conditions (temperature T), and the accepted safety factor.
\end{abstract}

The standardization of defects in welded metal structures, as a rule, is based on the methods of calculating their load-bearing capacity, taking into account the alleged failure mechanism during operation. In this case, the procedure for determining the permissible defect sizes is carried out proceeding from the realization of the case, which is the most unfavorable from the point of view of loss of the bearing capacity, corresponding to the minimum level of destructive stresses [1-3]. This approach to the standardization of defects contributes to a significant increase in the amount of repair work, which leads to an increase in the cost and timing of manufacturing or repair.

Recently, when assessing the load-carrying capacity of metal structures, widely used methods of calculation based on the application of interpolation-type criteria based on the principle of boundary interpolation. In accordance with this approach, the solution for intermediate states is represented in the form of interpolation relations between known boundary interpolations corresponding to two alternative mechanisms of fracture: brittle and viscous. The use of this approach allows, in our opinion, to carry out the rationing of

\footnotetext{
*Corresponding author: olgai3@mail.ru
} 
defects taking into account their location, sharpness at the top of the defects, as well as the properties of the base metal or welded joints.

At the heart of the proposed solution is one of the criteria of the interpolation type [4], taking into account the mixed nature of the destruction of metal structures during their operation

$$
\left(\frac{\Lambda_{i}}{\Lambda_{\rho}}\right)^{S}+\left(\frac{K_{I}}{K_{I C}(\rho)}\right)^{2 S} \leq 1
$$

where $\mathrm{s}$ is the interpolation parameter (as a rule, $\mathrm{s}=2$ ); $K_{I C}^{*}(\rho)$ is the critical stress intensity factor (the fracture toughness of the structural metal or welded joint); $K_{I}$ is the stress intensity factor, which can be represented in the following form [5]

$$
K_{I}=\sigma_{\text {ном }} \sqrt{\frac{\pi}{2}} l \cdot f_{K} \cdot f_{M} \cdot f_{\Pi л} \cdot f_{\rho},
$$

where $\sigma_{\text {ном }}$ the nominal stresses in the wall of the metal structure; $l$-size (depth) of the defect in the thickness direction of the metal structure $\mathrm{t} ; f_{K}$-correction for the thickness of the metal wall $\left(f_{K}=\sqrt{\sec \left(\frac{\pi}{2} \frac{l}{t}\right)}-\right.$ Federsen's amendment [5]); $f_{M}$ - correction for the location of the defect ( $f_{M}=1.12$ - for the surface defect, $f_{M}=1$ - for the internal defect, [5]) (Figure 1).

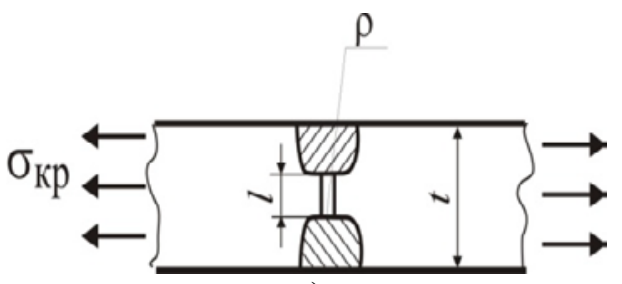

a)

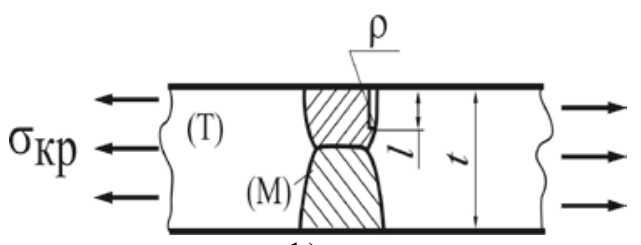

b)

Fig. 1. Fragment of a welded joint with an internal concentrator of finite radius (a) and surface nonfusion at the fusion boundary (b): (T) is the base metal; (M) - welded seam.

$f_{\text {ПЛ }}$ - correction for plasticity, taking into account the features of the formation of the plastic zone in the vicinity of the top of the defect.

For homogeneous welded joints in accordance with the approach [3], the correction $f_{\text {ПЛ }}$ can be obtained on the basis of the calculation scheme shown in Figure 2.

$$
f_{\text {ПЛ }}=\left[\frac{\cos ^{2} \theta}{1-\cos \theta}+\frac{1}{4}\left(\frac{1}{\cos \theta}-\cos 2 \theta\right)\right]^{-\frac{1}{4}},
$$

where $\theta$ is the slope angle of the wedge-shaped plasticity zone in the vicinity of the vertex of the defect (for the case of plane deformation $\theta= \pm \frac{\pi}{4}, f_{\Pi J}=0,84$ ). 


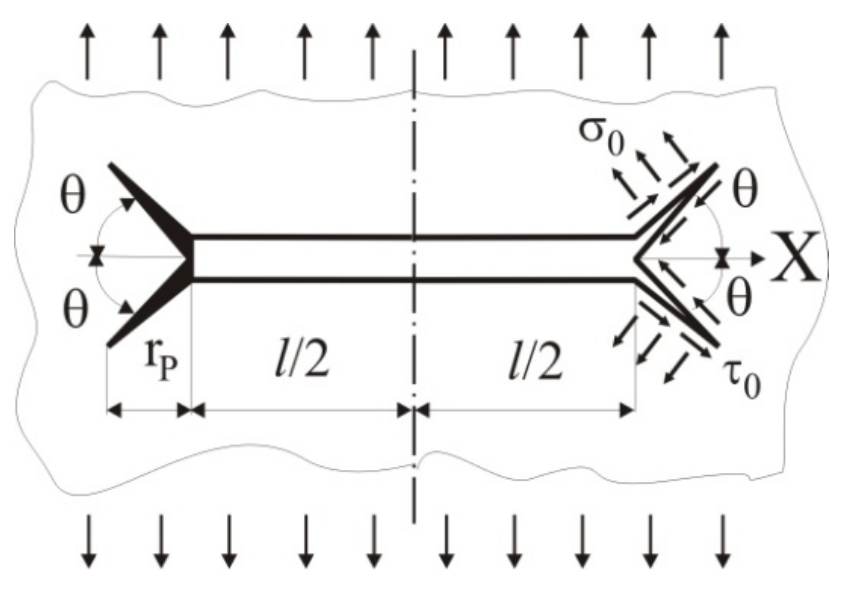

Fig. 2. Calculation scheme for the account of the plastic zone in the vicinity of the vertex of the defect

The correction for the sharpness of the defect $f \rho$ in determining the stress intensity factor $K_{I}$ was obtained on the basis of an adjustment of the Griffiths solution by taking into account the dependence of the size of the unloading zone above and below the defect on the radius value in the vicinity of its vertex [3]

$$
f_{\rho}=\left[1-\frac{(\pi-2)(2-\sqrt{2})}{\pi} \frac{2 \rho}{l}-\frac{(\pi-1)-(\pi-2)(2-\sqrt{2})}{\pi}\left(\frac{2 \rho}{l}\right)^{2}\right]^{1 / 4} .
$$

For crack-like defects such as faulty fusion, undercuts, with an internal concentrator of finite radius and and the like $f_{\rho} \approx 1$.

The critical stress intensity factor $K_{I C}$, also depends on the sharpness at the vertex of the defect and for the case under consideration can be determined taking into account the following relation [3]

$$
K_{I C}(p)=K_{I C} \cdot F_{\rho},
$$

where $F_{\rho}$ is the correction for the sharpness of the defect in determining $K_{I C}$

$$
\left\{\begin{array}{l}
F_{\rho}=\sqrt{\rho / \rho_{0}} ; \rho_{0}=0,08 r_{\rho} ; r_{\rho}=\frac{1}{6 \pi}\left(\frac{K_{I C}}{\beta \sigma_{6}}\right)^{2} ; \text { at } \rho>\rho_{0} \\
F_{\rho}=1, \text { at } \rho \leq \rho_{0}
\end{array}\right.
$$

The parameters in the left parenthesis of relation (1), $\Lambda \mathrm{i}$ and $\Lambda \mathrm{p}$, describe the boundary of the interpolation range corresponding to the viscous destruction of the compounds in question with a concentrator (defect).

The value of $\Lambda \mathrm{i}$, characterizing the level of accumulated damage in the vicinity of the vertex of the concentrator, was determined in accordance with [3.5], taking into account the effect of stress concentration in the vicinity of the vertex of the defect [6] 


$$
\Lambda_{i}=0,278 \frac{K_{1}^{2} \mathrm{~K}_{\sigma}^{2}}{\sigma_{\mathrm{T}} E \rho}
$$

where $\mathrm{K}_{\sigma}$ is the stress concentration coefficient in the vicinity of the vertex of the defect of finite radius [7].

$$
K_{\sigma}=1+2 \sqrt{1 / 2 \rho}
$$

The value of $\Lambda \mathrm{p}$, which characterizes the limiting stock of ductility of the metal of the construction or welded joint, was determined on the basis of the results of [8], according to this paper, the plasticity diagram can be represented as an exponential function $\Lambda_{\rho}=\Lambda_{0} \mathrm{e}^{-\alpha \Pi f\left(v_{\sigma}\right)}$ (where $\Lambda_{0}$ is the plasticity margin for pure shear, that is, for $\Pi=0$ ). In accordance with this, a relation was obtained that allows for the parameters of the approximated curve of material deformation $\sigma_{i}=\sigma_{\mathrm{T}}\left(E \frac{\varepsilon_{i}}{\sigma_{\mathrm{T}}}\right)^{m}: \mathrm{m}$ (index of hardening of the material), E and $\sigma \mathrm{T}$ (modulus of elasticity and yield stress) and parameters $\Pi$ and $v_{\sigma}$, determining the scheme and nature of the loading, to estimate the stock of ductility of the metal

$$
\Lambda_{\rho}=\frac{2 \mathrm{~m}}{1-\mathrm{m}^{2}} \mathrm{e}^{-\frac{\Pi}{2 \pi} f\left(\mathrm{~m}, \frac{\mathrm{E}}{\sigma_{\mathrm{T}}}, \nu_{\sigma}\right)}
$$

where

$$
f\left(\mathrm{~m}, \frac{\mathrm{E}}{\sigma_{\mathrm{T}}}, v_{\sigma}\right)=\ln \left(\frac{\mathrm{m}}{10 \pi} \frac{\mathrm{E}}{\sigma_{\mathrm{T}}}\left[1+\pi-\arccos \left(\frac{v_{\sigma}}{3}\right)\right]^{-2}\right) .
$$

The index of hardening of the material $\mathrm{m}$ that enters into this relation (10) can be determined from the known value of the limiting uniform strain $\varepsilon p$ [9]

$$
\varepsilon_{\rho}=\ln \left(\frac{1}{1-\psi}\right)
$$

Where $\psi$ is the relative narrowing of the material .

$$
m=\frac{\varepsilon_{\rho}}{2} .
$$

The value of $\Pi$, which determines the rigidity of the stress state in the most loaded part of the structure, depends on a number of factors [3]. In particular, in the vicinity of the vertex of a crack-like defect, under the conditions of plane deformation $\Pi=4.14$. Value $v_{\sigma}$ - an indicator of the form of the stress state in the pre-destruction zone (for the case of 
plane deformation, which is realized in the vicinity of the vertex of the concentrator, $\left.v_{\sigma}=0\right)$.

In accordance with the interpolation approach, based on the ratio (1), we can estimate the level of nominal stresses $\sigma_{\text {ном }}$ in the wall of the metal structure weakened by the defect

$$
\tilde{\sigma}=\frac{\sigma_{\text {ном }}}{\sigma_{\mathrm{T}}} \leq \frac{3,2}{f_{\mathrm{M}}} \sqrt{\frac{\rho / \mathrm{t}\left[\frac{\cos (\pi l / 2 t)}{l / t}\right]}{\frac{K_{\sigma}^{2}}{\Lambda_{p}} \cdot \frac{\sigma_{T}}{E}+0,015}}
$$

Figure 3 shows the curve $\sigma_{\text {ном }} / \sigma_{T}=\mathrm{f}(l / t)$, constructed taking into account the relations (2) - (12), characterizing the load-carrying capacity of welded joints made of steel ВСт.3пс (analogue: USA A570-36; Germany RSt37-2) (thickness $\mathrm{t}=20 \mathrm{~mm}$ by mechanized welding in shielding gas $(\mathrm{CO} 2)$ medium by a filler wire Св-08Г2C (analogue BÖHLER, Austria EML5; LINCOLN, USA Lincolnweld L50 SUPRA MID) diameter $2 \mathrm{~mm}$.

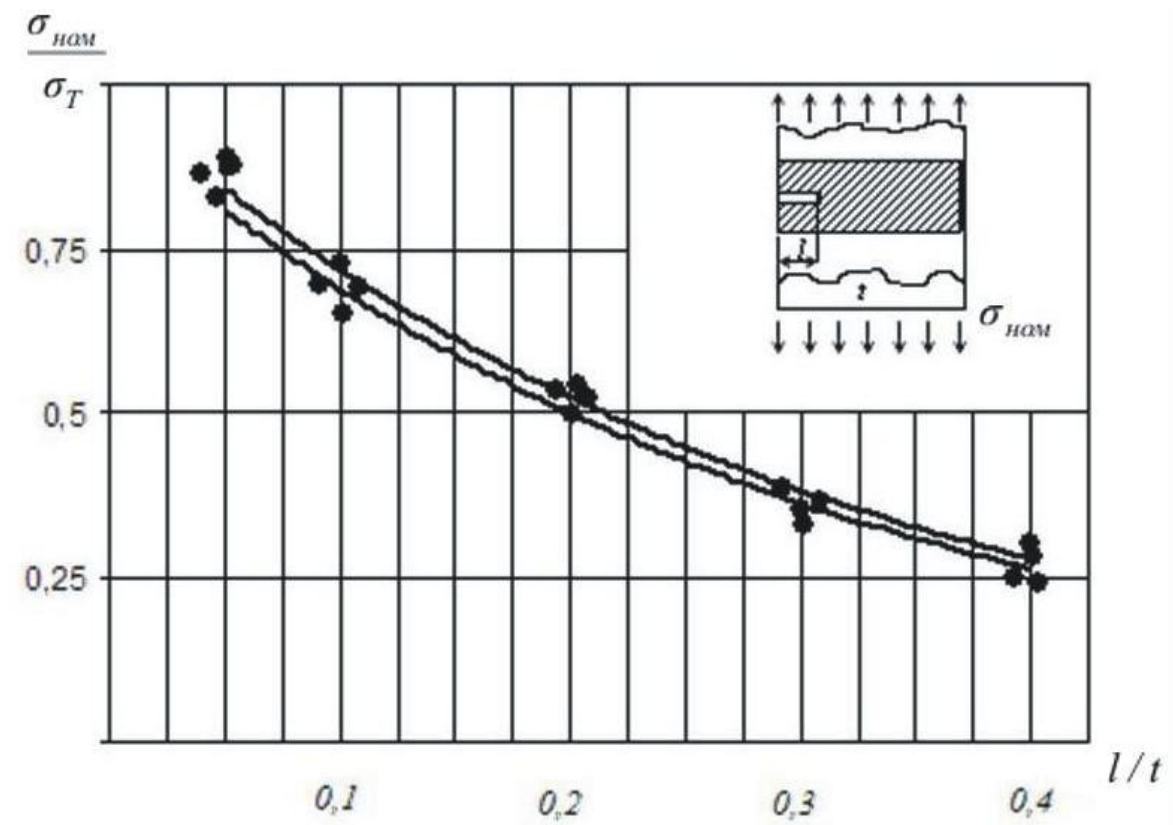

Fig. 3. Comparison of calculated and experimental data on the evaluation of the strength of welded joints with surface defects from steel ВСт.3пс:

- the relation (12), - experiment at $-\mathrm{T}=213 \mathrm{~K}$.

Experimental data obtained during testing of welded flat samples with a surface defect located in the central plane of the welded seam are also presented here. The defect was applied mechanically (the radius at the vertex $\rho=0.1 \ldots 0.14 \mathrm{~mm}$, the depth of the defect $l=1 \ldots 8 \mathrm{~mm} \quad(l / t=0,05 \ldots 0,4)$. Tests of the samples were carried out at a temperature of $\mathrm{T}=213 \mathrm{~K}\left(\mathrm{~T}=-60^{\circ} \mathrm{C}\right)$. The strength characteristics of the weld metal at the test temperature were respectively $\sigma_{\mathrm{B}}=400 \mathrm{MPa}$ and $\sigma_{\mathrm{T}}=300 \mathrm{MPa}$.

As can be seen, the use of interpolation-type criteria in assessing the bearing capacity of welded joints with a defect provides an acceptable convergence of the calculated and experimental values of the strength of welded joints with a surface defect. 
The resulting relation (12) allows us to estimate the range of permissible defect sizes [0; $\left.(l / t)_{\partial}\right]$, which do not lead to a decrease in the operational loads acting on welded metal structures during their operation, based on ensuring the required level of permissible stresses $[\sigma]=\sigma_{\mathrm{T}} / \mathrm{n}$ (where $\mathrm{n}$ is the safety factor for the yield strength, $\mathrm{n}=1.3 \ldots 1.6$ ). For example, for the welded joints of steel VS.sub.3p.s under the conditions of operation of metal structures $\left(\left(T=-600\right.\right.$ C.) and the level of permissible stresses $[\sigma]=0,66 \sigma_{\mathrm{T}}(n=1,5)$, the range of permissible sizes of surface defects [0; $(1 / \mathrm{t})$ д] with a radius at the vertex $(0.1 \ldots$ $0.14 \mathrm{~mm}$ ) is in the interval $[0 ; 0,1]$ (see Figure 3).

Mathematically allowable range of defect sizes for the case under consideration can be represented by the following relation

$$
0 \leq\left(\frac{l}{t}\right)_{\partial} \leq 10 \frac{\Lambda_{\mathrm{p}}}{f_{\mathrm{M}}^{2}} \frac{E}{\sigma_{T}} \frac{\rho}{t} \frac{n^{2}}{K_{\sigma}^{2}} .
$$

\section{References}

1. I.P. Belokur, On the criteria for evaluating the defects of welded joints, Automatic welding, v. 5, pp. 30-33(1975)

2. I.I. Makarov, Criteria for assessing technological defects in welded structures, Welding production, v. 12, pp. 9-1 (1975)

3. M.V. Shakhmatov, V.V. Erofeev, V.V. Kovalenko, Performance and non-destructive testing of welded joints with defects (CSTI, Chelyabinsk 2000).

4. E.M. Morozov, Two-criteria approaches in the mechanics of fracture, Problems of Strength, v. 10, pp. 103-108 (1985)

5. N.A. Makhutov, Resistance of structural elements to brittle fracture (M ., Mechanical Engineering, 1973)

6. V. V. Erofeev, O. A. Grebenshchikova, on the peculiarities of the use of the principle of boundary interpolation in the normalization of defects in welded metal structures, Proceedings of the YII Ural scientific-practical conference: welding. Renovation. Tribology (2015)

7. A. Kelly, High-strength materials, (M ., Publishing house "Mir", 1976)

8. V.V. Erofeev M.V. Shakhmatov, V.V. Kovalenko et al., Estimation of the resource of plasticity of the metal according to certification data (Materials of the report of the XXI Russian School on the problems of science and technology, Miass 2001)

9. A.D. Tomlenov, Theory of plastic deformation of metals ( M., Metallurgy, 1972) 